\title{
A Tilting Approach to Ranking Influence
}

\author{
Marc G. Genton ${ }^{1}$ and Peter Hall ${ }^{2}$
}

September 30, 2014

\begin{abstract}
We suggest a new approach, applicable for general statistics computed from random samples of univariate or vector valued or functional data, to assessing the influence that individual data have on the value of a statistic, and to ranking the data in terms of that influence. Our method is based on, first, perturbing the value of the statistic by "tilting," or reweighting, each data value, where the total amount of tilt is constrained to be the least possible, subject to achieving a given small perturbation of the statistic; and then, taking the ranking of the influence of data values to be that which corresponds to ranking the changes in data weights. It is shown, both theoretically and numerically, that this ranking does not depend on the size of the perturbation, provided the perturbation is sufficiently small. That simple result leads directly to an elegant geometric interpretation of the ranks; they are the ranks of the lengths of projections of the weights onto a "line" determined by the first empirical principal component function in a generalised measure of covariance. To illustrate the generality of the method we introduce and explore it in the case of functional data, where (for example) it leads to generalised boxplots. The method has the advantage of providing an interpretable ranking that depends on the statistic under consideration. For example, the ranking of data, in terms of their influence on the value of a statistic, is different for a measure of location and for a measure of scale. This is as it should be; a ranking of data in terms of their influence should depend on the manner in which the data are used. Additionally, the ranking recognises, rather than ignores, sign, and in particular can identify left- and righthand "tails" of the distribution of a random function or vector.
\end{abstract}

Some key words: Band depth; Data weights; Functional boxplot; Functional data; Image data; Outlier; Robustness.

Short title: Tilting and Ranking Influence

\footnotetext{
${ }^{1}$ CEMSE Division, King Abdullah University of Science and Technology, Thuwal 23955-6900, Saudi Arabia. E-mail: marc.genton@kaust.edu.sa

Genton's research was partially supported by a grant from the University of Melbourne as an Honorary Fellow.

${ }^{2}$ University of Melbourne and University of California, Davis

E-mail: halpstat@ms.unimelb.edu.au
} 


\section{Introduction}

The analysis of functional data has received sustained attention in recent years due to the increasing availability and collection of observations that arise as functions or images. Data of this type arise in many disciplines, and include growth curves in biology, climate variables from networks of monitors or from climate model outputs in geosciences, profiles derived by monitoring manufacturing processes, to name a few; see the books by Ramsay and Silverman (2005), Ferraty and Vieu (2006), Ramsay et al. (2009), and references therein.

Classical statistical methods based on ranks can be extended to the functional setting. To this end, López-Pintado and Romo (2009) introduced a notion of band depth. It yields an ordering of a sample of functional data from the center outwards and therefore defines a measure of the centrality or outlyingness of an observation. For instance, the median function, or a trimmed mean function, can be defined for robust statistical analysis, and functional boxplots (Sun and Genton, 2011, 2012a) or surface boxplots (Genton et al., 2014) can be constructed for visualization and outlier detection. Sun and Genton (2012b) proposed a functional median polish algorithm, based on using a band depth functional median, to fit robustly a functional analysis of variance model. Sun et al. (2012) derived a fast algorithm to compute band depth for functional data and LópezPintado et al. (2014) introduced simplicial band depth for multivariate functional data. Yu et al. (2012) proposed a test for functional outlier detection, founded on functional principal components. Hyndman and Shang (2010) applied functional principal component analysis to a robust covariance matrix in order to detect outliers. The latter two approaches have the drawback of being basis-dependent. Genton and Ruiz-Gazen (2010) introduced another approach to defining and visualizing influential observations in dependent data based on additive data perturbations.

In this paper we suggest a new, widely applicable approach to ranking influential data, based on data tilting. Relative to previously considered methods, this technique has the advantages 
of being basis-independent, of ranking data according to their influence on specific statistics (e.g. our rankings in the context of location estimation can be quite different from those for scale estimation), of allowing the identification of "tails" in distributions of random functions or vectors, and of being applicable in an exceptionally wide range of settings. For instance, the method can be employed to identify influential data in random samples, no matter whether those data are univariate or vector valued or function valued, for many different estimator types. In the case of functional data, the functions can have almost arbitrary arguments, of any dimension, and the statistics being considered can involve tuning parameters, in which case the rankings reflect those parameters. For brevity our notation and our examples will address the functional data case.

Data tilting involves replacing uniform weights on the data by more general weights. It can be employed to render parametric procedures more robust, or to produce a natural ordering of the data in terms of their contributions to the fit of a model (Choi et al., 2000). The use of tilting for assessing robustness, or the influence of particular data values, has been investigated by Hall and Presnell (1999b), Critchley and Marriott (2004), Lazar (2005), and Camponovo and Otsu (2012), among others. Many applications of tilting and perturbation arguments to contemporary, nonparametric statistical problems have been developed, including those of Hall and Presnell (1999a), Critchley et al. (2001), Hall and Yao (2003), Bravo (2005), Hall et al. (2009), Xu and Phillips (2011), and the vast literature on empirical likelihood.

In this paper we use tilting to assign a rank to each observation, for example each data function. We show that this ranking admits a simple, general interpretation in terms of projections of functions of the data onto the space spanned by an empirical eigenfunction corresponding to the largest eigenvalue, where the linear transformation that determines the eigenvalue and eigenfunction is determined by the statistic under investigation. This interpretation is not asymptotic 
in terms of sample size, and so is available, and relevant, even for small sample sizes.

The remainder of this paper is organized as follows. In Section 2 we give a general definition of ranking based on tilting, and introduce our methodology. We also outline theory associated with this approach. The methodology and its numerical properties are illustrated through a real data example in Section 3. The proof of our main result is relegated to the Appendix. The data and code can be obtained at stsda.kaust.edu.sa/Pages/Software.aspx

\section{Methodology}

\subsection{Examples of tilting}

Assume we observe data $X_{1}, \ldots, X_{n}$, supported in a region $\mathcal{R}$. For definiteness we take the data to be functions, but it will be appreciated that our methodology is available more generally. The data $X_{i}$ s may be paired with other data $Y_{i}$, for example response variables in a regression problem, and in this case the pairs $\left(X_{i}, Y_{i}\right)$ are assumed to be distributed as $(X, Y)$. In the present subsection we shall discuss tilted versions of four statistics $\widehat{\omega}$, defined in (1), (2), (4) and (5). There, $W$ is a kernel function, $h$ a bandwidth, $t \in \mathcal{R}$ in (1) and (4), $t=\left(t_{1}, t_{2}\right)$ in $(2)$ is

a vector in $\mathcal{R} \times \mathcal{R}, v=(t, u) \in \mathcal{R} \times \mathbb{R}$ in (5), and the quantities $\hat{\theta}_{j}$ and $\hat{\phi}_{j}$ will be defined and discussed below (6):

$$
\begin{aligned}
\widehat{\omega}(t) & =\bar{X}(t)=\frac{1}{n} \sum_{i=1}^{n} X_{i}(t), \\
\widehat{\omega}(t)= & \widehat{K}\left(t_{1}, t_{2}\right) \equiv \frac{1}{n} \sum_{i=1}^{n}\left\{X_{i}\left(t_{1}\right)-\bar{X}\left(t_{1}\right)\right\}\left\{X_{i}\left(t_{2}\right)-\bar{X}\left(t_{2}\right)\right\} \\
& =\sum_{j=1}^{\infty} \hat{\theta}_{j} \hat{\phi}_{j}\left(t_{1}\right) \hat{\phi}_{j}\left(t_{2}\right) \\
\widehat{\omega}(t)= & \hat{\phi}_{j}(t), \quad\left[\sum_{i=1}^{n} Y_{i} W\left\{\frac{X_{i}(t)-u}{h}\right\}\right] /\left[\sum_{i=1}^{n} W\left\{\frac{X_{i}(t)-u}{h}\right\}\right] .
\end{aligned}
$$


We consider $\widehat{\omega}$ to be an estimator of a quantity $\omega$. In $(1), \widehat{\omega}(t)$ is the empirical mean of the functions $X_{i}$, evaluated at $t$, and estimates $\omega(t)=E\{X(t)\}$; and $t \in \mathcal{R}$. In $(2), \widehat{\omega}(t)$ denotes an estimator of the covariance function, $\omega=K$, defined by

$$
K\left(t_{1}, t_{2}\right)=\operatorname{cov}\left\{X\left(t_{1}\right), X\left(t_{2}\right)\right\}=\sum_{j=1}^{\infty} \theta_{j} \phi_{j}\left(t_{1}\right) \phi_{j}\left(t_{2}\right)
$$

The singular value decompositions at (3) and (6) are the versions, for functional data, of empirical and theoretical, respectively, principal component covariance expansions expressed in terms of (eigenvalue, eigenfunction) pairs $\left(\hat{\theta}_{j}, \hat{\phi}_{j}\right)$ and $\left(\theta_{j}, \phi_{j}\right)$, and ordered so that $\hat{\theta}_{1} \geq \hat{\theta}_{2} \geq \ldots$ and $\theta_{1} \geq \theta_{2} \geq \ldots$ In $(4), \omega=\phi_{j}$. In (5), $\widehat{\omega}(v)$ is an estimator of the conditional mean, $\omega(v)=E\{Y \mid X(t)=u\}$. Result (6) holds, with convergence in mean square, provided that $\int_{\mathcal{R}} E\left\{X(t)^{2}\right\} d t<\infty$, and similarly (3) holds, with convergence in the same sense, as long as each data function is square-integrable.

Let $p=\left(p_{1}, \ldots, p_{n}\right)$ denote an $n$-vector whose components are nonnegative and satisfy $\sum_{i} p_{i}=1$. (Such a vector is often referred to in the literature as a "multinomial distribution.") Let $\widehat{\omega}_{p}$ denote the version of $\widehat{\omega}$ when each data value $X_{i}$, or the pair $\left(X_{i}, Y_{i}\right)$, is weight by the respective value of $p_{i}$, rather than by $n^{-1}$. We say that $X_{i}$ has been tilted using $p$. In the examples at (1), (2), (4) and (5) we have, respectively,

$$
\begin{aligned}
& \widehat{\omega}_{p}(t)= \bar{X}_{p}(t)=\sum_{i=1}^{n} p_{i} X_{i}(t) \\
& \widehat{\omega}_{p}(t)= \widehat{K}_{p}\left(t_{1}, t_{2}\right)=\sum_{i=1}^{n} p_{i}\left\{X_{i}\left(t_{1}\right)-\bar{X}_{p}\left(t_{1}\right)\right\}\left\{X_{i}\left(t_{2}\right)-\bar{X}_{p}\left(t_{2}\right)\right\} \\
&=\sum_{j=1}^{\infty} \hat{\theta}_{p j} \hat{\phi}_{p j}\left(t_{1}\right) \hat{\phi}_{p j}\left(t_{2}\right) \\
& \widehat{\omega}_{p}(t)= \hat{\phi}_{p j}(t), \quad \\
& \widehat{\omega}_{p}(v)=\left[\sum_{i=1}^{n} p_{i} Y_{i} W\left\{\frac{X_{i}(t)-u}{h}\right\}\right] /\left[\sum_{i=1}^{n} p_{i} W\left\{\frac{X_{i}(t)-u}{h}\right\}\right] .
\end{aligned}
$$


If we write

$$
p_{0}=\left(n^{-1}, \ldots, n^{-1}\right)
$$

for the uniform distribution on $n$ points, then the quantities $\widehat{\omega}$, defined at (1), (2), (4) and (5), are denoted equivalently by $\widehat{\omega}_{p_{0}}$. The $L_{2}$ distance between the tilted and nontilted forms of $\widehat{\omega}$ is

$$
d_{1}(p)=\int_{\mathcal{J}}\left\{\widehat{\omega}_{p}(v)-\widehat{\omega}(v)\right\}^{2} d v=\int_{\mathcal{J}}\left\{\widehat{\omega}_{p}(v)-\widehat{\omega}_{p_{0}}(v)\right\}^{2} d v,
$$

where $\mathcal{J}$ is a suitable set; for example, it would be taken equal to $\mathcal{R}$ in the first and third examples, to $\mathcal{R} \times \mathcal{R}$ in the second example, and to a subset of $\mathcal{R} \times \mathbb{R}$ in the fourth.

\subsection{Methodology based on tilting}

Let $p=\left(p_{1}, \ldots, p_{n}\right)$ represent a general multinomial distribution on $n$ points, introduced in Section 2.1; let $p_{0}$ be the uniform probability distribution, defined at (10); let $d_{1}(p)$, at (11), denote the distance between the tilted and untitled forms of the statistic $\widehat{\omega}$; and measure the distance between $p$ and $p_{0}$ using the criterion

$$
d_{2}(p)=\sum_{i=1}^{n} p_{i} \log \left(n p_{i}\right)
$$

Given $\epsilon>0$, choose $p$ to minimise $d_{2}(p)$ subject to

$$
d_{1}(p)=\epsilon^{2}
$$

Minimising $d_{2}(p)$ is equivalent to maximising a measure of entropy, or equivalent to minimising a measure of Kullback-Leibler divergence. The resulting value of $p$, denoted by $\left(p_{1}(\epsilon), \ldots, p_{n}(\epsilon)\right)$, is our tilted form of the discrete uniform distribution. We rank the values of $p_{i}(\epsilon)$, obtaining

$$
p_{\hat{\imath}_{1}}(\epsilon) \leq \ldots \leq p_{\hat{\imath}_{n}}(\epsilon)
$$


say. This induces an ordering of the data set, and we express that ordering as $\hat{\imath}_{1}(\epsilon) \succeq \ldots \succeq \hat{\imath}_{n}(\epsilon)$.

The distance measure at (12) is one of the power divergence distances (see e.g. Cressie and Read, 1984), and has advantages over other contenders in that it allows one or more of the $p_{i} \mathrm{~s}$ to equal 0 , and leads automatically to nonnegative $p_{i}$ s without requiring an algorithm such as quadratic programming to ensure that property.

Our ranking of influence recognises, rather than ignores, sign. In particular, if $\widehat{\omega}$ is a mean then at one end of the sequence $\hat{\imath}_{1}, \ldots, \hat{\imath}_{n}$ the indices identify data values that are having a relatively large positive influence, while at the other end they are having a relatively large negative influence. This issue motivates our preference for the ranking $\hat{\imath}_{1} \succeq \ldots \succeq \hat{\imath}_{n}$ rather than $\hat{\imath}_{n} \succeq \ldots \succeq \hat{\imath}_{1}$, because the former acknowledges the positive role played by $X_{\hat{\imath}_{1}}$, and the negative role of $X_{\hat{\imath}_{n}}$. This is transparent in the case of a scalar mean, and similar arguments can be given in the case of function valued data and other statistics $\widehat{\omega}$, where our approach allows identification of left- and right-hand "tails" of general distributions. See, for example, the discussion in Section 3.2.

If the distribution of the data is continuous then, in the limit as $\epsilon \downarrow 0$, the inequality signs in (14) are strict with probability 1. That is, for each strictly positive, deterministic choice of $\epsilon$, $P\left\{p_{\hat{\imath}_{1}}(\epsilon)<\ldots<p_{\hat{\imath}_{n}}(\epsilon)\right\}=1$. Therefore the ordering is strict; rather than $\hat{\imath}_{1}(\epsilon) \succeq \ldots \succeq \hat{\imath}_{n}(\epsilon)$, we have:

$$
\hat{\imath}_{1}(\epsilon) \succ \ldots \succ \hat{\imath}_{n}(\epsilon)
$$

Moreover, as we shall argue in section 2.3, the strict ranking in (15) remains valid in the limit as $\epsilon \downarrow 0$.

Given that we have tilted $p_{0}$ to $p=p(\epsilon)=\left(p_{1}(\epsilon), \ldots, p_{n}(\epsilon)\right)$, we rank the values $p_{1}(\epsilon), \ldots, p_{n}(\epsilon)$, obtaining the inequalities at (14). We assert that $X_{\hat{\imath}_{1}}, \ldots, X_{\hat{\imath}_{n}}$ is an ordering of $X_{1}, \ldots, X_{n}$ in decreasing order of their immediate influence on the value of $\widehat{\omega}$, respecting the sign of influence, 
and we express the corresponding ordering of the indices $1, \ldots, n$ as $\hat{\imath}_{1}(\epsilon) \succeq \ldots \succeq \hat{\imath}_{n}(\epsilon)$.

Potentially, the application of these ideas is hindered by the fact that $\hat{\imath}_{1}(\epsilon), \ldots, \hat{\imath}_{n}(\epsilon)$ depend on $\epsilon$, since an appropriate choice of $\epsilon$ may require delicate empirical methods. However, under mild assumptions, the quantities $\hat{\imath}_{j}(\epsilon)$ have proper, well-defined limits as $\epsilon \downarrow 0$. Moreover, the process of convergence to the limit here has several statistically attractive properties: (1) The entire sequence $\hat{\imath}_{1}(\epsilon), \ldots, \hat{\imath}_{n}(\epsilon)$ has a well-defined, joint limit as $\epsilon \downarrow 0$; (2) The ordering of the limiting values $\hat{\imath}_{1}(0), \ldots, \hat{\imath}_{n}(0)$ has attractive, statistically interpretable features, expressible in terms of generalised principal components and which we discuss in the next section; (3) If the distribution of the random function $X$ is continuous then, with probability 1 , there are no ties in the limiting ordering; and (4) In the setting of (3), the ordering is identical to $\hat{\imath}_{1}(\epsilon) \succeq \ldots \succeq \hat{\imath}_{n}(\epsilon)$ for all sufficiently small $\epsilon$, including $\epsilon=0$, and so is straightforward to identify simply by considering successively smaller values of $\epsilon$.

Using the ideas in this paper as a foundation, a number of directions might be pursued in the future. First, breakdown points could be considered, although they are arguably beyond the scope of the present paper, not least because we are developing a general approach to ranking for general statistics for general types of data. In comparison, the study of breakdown points usually demands a much narrower focus. For example, in the case of location estimation it is often necessary to assume that the sampled distribution is symmetric about its centre, whereas quite different models are needed for estimators of other quantities. Secondly, one might try to measure and compare the "outlyingness" of outlying data. For that problem we can offer at present no better suggestion than to compute $\widehat{\omega}$ with the outlying value removed, and determine in that way just how much leverage the removed value has. Thirdly, motivated by the theoretical results we shall discuss in section 2.3, one could estimate higher-order principal component functions that correspond to a general statistic $\widehat{\omega}$, and use that analysis to gain insight into higher-order 
aspects of rankings of influence. However, this approach is likely to be challenged by the sheer complexity of those high-order features.

\subsection{Limit of the ranking as $\epsilon \downarrow 0$}

First we state our assumptions. We assume that, as $\epsilon \downarrow 0$, we can write

$$
\widehat{\omega}_{p}-\widehat{\omega}=\sum_{i=1}^{n}\left(p_{i}-n^{-1}\right) \Delta_{i}+O\left(\epsilon^{2}\right)
$$

and

$$
\frac{1}{2} \frac{\partial}{\partial p_{j}} \int_{\mathcal{R}}\left(\widehat{\omega}_{p}-\widehat{\omega}\right)^{2}=\sum_{i=1}^{n}\left(p_{i}-n^{-1}\right) \int_{\mathcal{R}} \Delta_{i} \Delta_{j}+A_{j}+O\left(\epsilon^{3}\right),
$$

where the integral is over the support, $\mathcal{R}$, of $\widehat{\omega}_{p}$ and $\widehat{\omega}$, which is assumed to be compact; and $\Delta_{i}$ denotes an approximation to the first derivative of $\widehat{\omega}_{p}$ with respect to $p_{i}$ (the approximation is accurate up to a remainder of order $\left.\epsilon^{2}\right)$. We ask too that, as $\epsilon \downarrow 0$,

$$
\sum_{i=1}^{n} \Delta_{i}=\sum_{i=1}^{n} A_{i}=0, \quad \Delta_{i}=O(1), \quad A_{i}=O\left(\epsilon^{2}\right) \quad \text { for each } i
$$

The quantities $\Delta_{i}$ may depend on $p$, although only negligibly so; they will satisfy

$$
\Delta_{i}=\Delta_{i}^{0}+O(\epsilon) \text { for each } i
$$

as $\epsilon \downarrow 0$, where $\Delta_{i}^{0}$ depends only on the data.

To convey intuition about these assumptions we mention that (16) asserts that, to first order, $\widehat{\omega}_{p}-\widehat{\omega}$ is linear in perturbations $p_{i}-n^{-1}$ of the data weights, with a quadratic remainder, and (17) essentially follows from (16) on squaring both sides and integrating; see the discussion three paragraphs below. The first part of (18) reflects the fact that the $p_{i}$ s are related by the formula $\sum_{i} p_{i}=1$, and in particular are not independent variables; and the second and third parts of (18) stipulate that the first, second and third terms on the right-hand side of (17) are of orders $\epsilon$, 
$\epsilon^{2}$ and $\epsilon^{3}$, respectively. Condition (19) asserts only that $\Delta_{i}$, which as (18) states is of order 1 , does not depend on $\epsilon$ to first order. These assumptions are satisfied widely, as will be shown through examples later in this subsection.

Define

$$
\widehat{M}\left(t_{1}, t_{2}\right)=\frac{1}{n} \sum_{i=1}^{n} \Delta_{i}\left(t_{1}\right) \Delta_{i}\left(t_{2}\right),
$$

for $t_{1}, t_{2} \in \mathcal{R}$. We also write $\widehat{M}$ for the operator itself: If $\chi$ is a function then

$$
(\widehat{M} \chi)\left(t_{1}\right)=\int_{\mathcal{R}} \widehat{M}\left(t_{1}, t_{2}\right) \chi\left(t_{2}\right) d t_{2}
$$

Although $\widehat{M}$ may depend on $p$, through the quantities $\Delta_{i}$, in view of (19) this dependence becomes negligible as $\epsilon \downarrow 0$. Let $\widehat{M}^{0}$ denote the limit of $\widehat{M}$ as $\epsilon$ decreases. Then we can write

$$
\widehat{M}^{0}\left(t_{1}, t_{2}\right)=\frac{1}{n} \sum_{i=1}^{n} \Delta_{i}^{0}\left(t_{1}\right) \Delta_{i}^{0}\left(t_{2}\right)=\sum_{j=1}^{\infty} \hat{\alpha}_{j} \hat{\beta}_{j}\left(t_{1}\right) \hat{\beta}_{j}\left(t_{2}\right)
$$

representing a conventional singular value decomposition of the positive definite operator with kernel $\widehat{M}^{0}$, having (eigenvalue, eigenfunction) pairs denoted by $\left(\hat{\alpha}_{j}, \hat{\beta}_{j}\right)$. It is assumed that the terms in the second series in $(20)$ are ordered so that $\hat{\alpha}_{1} \geq \hat{\alpha}_{2} \geq \ldots$

There is, of course, a simple relationship between (16) and (17). Indeed, since $\Delta_{i}$ equals the first derivative of $\widehat{\omega}_{p}-\widehat{\omega}$ with respect to $p_{i}$, up to a remainder of order $\epsilon^{2}$; and since, by (16), $\widehat{\omega}_{p}-\widehat{\omega}$ equals $\sum_{i}\left(p_{i}-n^{-1}\right) \Delta_{i}$, up to a remainder of the same order; then, differentiating under the integral sign, it is clear that, up to a remainder of order $\epsilon^{2}$, half the value of the first derivative of $\int\left(\widehat{\omega}_{p}-\widehat{\omega}\right)^{2}$, with respect to $p_{j}$, should equal

$$
\int\left\{\sum_{i=1}^{n}\left(p_{i}-n^{-1}\right) \Delta_{i}\right\} \Delta_{j}
$$

This is just the first term on the right hand side of (17). The second term there, i.e. $A_{j}$, represents the dominant contributions of order $\epsilon^{2}$. Therefore (17) simply confirms the influence of (16) on 
the derivative of $\int\left(\widehat{\omega}_{p}-\widehat{\omega}\right)^{2}$. The term $A_{j}$ vanishes in a number of important examples.

The orders of magnitude of remainder terms, above and in the work below, and also in the proof of the theorem in the Appendix, are to be interpreted as holding as $\epsilon \downarrow 0$ for fixed $n$, in fact for a fixed sample. They are not asymptotic in $n$.

To illustrate these assumptions we consider instances where $\widehat{\omega}$ is defined by (1) or (2). In the first of these cases, $\Delta_{i}=X_{i}-\bar{X}$ and $A_{i} \equiv 0$, where these random functions are from $\mathcal{R}$ to the real line. Here $\Delta_{i}^{0}$, in (19), is identical to $\Delta_{i}$. In the case of (2),

$$
\Delta_{i}\left(t_{1}, t_{2}\right)=\delta_{2 i}\left(t_{1}, t_{2}\right)-\delta_{1 i}\left(t_{1}\right) \xi\left(t_{2}\right)-\delta_{1 i}\left(t_{2}\right) \xi\left(t_{1}\right)
$$

and again $A_{i} \equiv 0$, where $t_{1}, t_{2} \in \mathcal{R}, \delta_{1 i}=X_{i}-\bar{X}, \delta_{2 i}=Z_{i}-\widehat{K}, \xi=\bar{X}_{p}-\bar{X}$ and

$$
Z_{i}\left(t_{1}, t_{2}\right)=\left\{X_{i}\left(t_{1}\right)-\bar{X}\left(t_{1}\right)\right\}\left\{X_{i}\left(t_{2}\right)-\bar{X}\left(t_{2}\right)\right\}
$$

Although the quantities $\Delta_{i}$, in (22), depend on $p$, they enjoy property (19) with $\Delta_{i}^{0}=\delta_{2 i}$.

Likewise, the nonparametric regression example at (5) satisfies (16)-(18). This indicates that the usefulness of our approach to ranking the influence of data extends well beyond the context of functional data analysis, where inference is typically semiparametric. Indeed, the example at (5) can be viewed as one of nonparametric regression, particularly if we fix $t$ and take the region $\mathcal{R}$ to be a subset of the real line, reflecting only the values taken by $u$ in (5), rather than to be a subset of $\mathcal{I} \times \mathbb{R}$ (if the random functions are defined on $\mathcal{I}$ ). The theorem below is valid in settings where the data are not just functions $X_{1}, \ldots, X_{n}$, but functions with other quantities adjoined, as in the paired data $\left(X_{1}, Y_{1}\right), \ldots,\left(X_{n}, Y_{n}\right)$; and also more complex settings where the $i$ th data "point" is actually a sample. The essential ingredient is that there should exist an analogue of integration, so that the operator $\widehat{M}$ at (20) can be defined.

Recall that the ordering $\hat{\imath}_{1}(\epsilon) \succeq \ldots \succeq \hat{\imath}_{n}(\epsilon)$ was introduced in Section 2.1, that the perturba- 
tion $\Delta_{i}^{0}$ was introduced at (19), that $\mathcal{R}$ denotes the support of the random functions, and that the eigenvalues $\hat{\alpha}_{j}$ and eigenvectors $\hat{\beta}_{j}$ are as at (21). When interpreting the theorem it helps if we note that $\hat{\beta}_{1}$ and $-\hat{\beta}_{1}$ are both eigenfunctions corresponding to the largest eigenvalue, $\hat{\alpha}_{1}$, and are indistinguishable in terms of that definition. Intuition behind (16)-(19) is given in the paragraph immediately below (19).

Theorem. If (16)-(19) hold, and if $\hat{\alpha}_{1}>\hat{\alpha}_{2}$, then, with probability 1, the limit as $\epsilon \downarrow 0$ of the ranking $\hat{\imath}_{1}(\epsilon) \succeq \ldots \succeq \hat{\imath}_{n}(\epsilon)$ is well defined, and is in fact the ordering $\hat{\imath}_{1} \succeq \ldots \succeq \hat{\imath}_{n}$ given by

$$
\int_{\mathcal{R}} \hat{\beta}_{1} \Delta_{\hat{\imath}_{1}}^{0} \geq \ldots \geq \int_{\mathcal{R}} \hat{\beta}_{1} \Delta_{\hat{\imath}_{n}}^{0}
$$

for one of the choices $\pm \hat{\beta}_{1}$.

In summary, the theorem asserts that the limit, as $\epsilon \downarrow 0$, of the ordering of $X_{1}, \ldots, X_{n}$ in terms of their influence on $\widehat{\omega}$, is identical to the ordering of the projections of the perturbations $\Delta_{1}^{0}, \ldots, \Delta_{n}^{0}$ onto the univariate space spanned by the first empirical principal component function for $\widehat{M}^{0}$. Since that function explains the greatest amount possible, for a single function, of the variability of the empirical distribution of perturbations corresponding to respective data functions $X_{i}$ (or data pairs $\left(X_{i}, Y_{i}\right)$ ), then this result enhances the intuitive appeal of assessing influence in terms of tilting.

To appreciate the implications of the theorem it is helpful to consider in detail the case $\widehat{\omega}=\bar{X}$, the sample mean for functional data. There the theorem asserts that the limit, as $\epsilon \downarrow 0$, of the ordering of $X_{1}, \ldots, X_{n}$ in terms of their influence, is identical to the ordering of the projections of $X_{1}, \ldots, X_{n}$ onto the univariate space spanned by the first empirical principal component function for $\widehat{K}$. In this case, $\Delta_{i}=X_{i}-\bar{X}$ and $\hat{\beta}_{1}=\hat{\phi}_{1}$, the latter introduced at (3), and therefore the 
ranking at (23) is identical to:

$$
\int_{\mathcal{R}} \hat{\phi}_{1} X_{\hat{\imath}_{1}}>\ldots>\int_{\mathcal{R}} \hat{\phi}_{1} X_{\hat{\imath}_{n}}
$$

Since $\hat{\phi}_{1}$ explains the greatest amount possible, for a single function, of the variability of the empirical distribution of the dataset $X_{1}, \ldots, X_{n}$, then the ranking at (24) is particularly reasonable.

For a general statistic $\widehat{\omega}$ the role of $X_{i}$ in the ranking, for example in (24), is replaced by $\Delta_{i}$, but the index $i$ still relates directly to the $i$ th observation, for example the $i$ th function $X_{i}$ or the $i$ th data pair $\left(X_{i}, Y_{i}\right)$. Perturbations of data weights results in eigenvalue estimators changing, and can lead to ties occurring among those quantities where they did not exist previously. That can result in difficulty identifying principal component functions. However, under the continuity assumption discussed in section 2.2, the probability of this occurring in the case $\epsilon=0$ is zero, and so it is not a problem at least in theory. We have not noticed it in numerical work.

\section{Numerical Properties}

\subsection{Computational aspects}

Assume we observe functional data $X_{1}\left(t_{k}\right), \ldots, X_{n}\left(t_{k}\right)$ at $m$ points $t_{1}, \ldots, t_{m}$ on the interval $\mathcal{I}$, which here plays the role of $\mathcal{R}$. At first sight, the tilting procedure described in Section 2 may seem to require solving an optimization problem of dimension $n$ in order to obtain the weights $p_{1}, \ldots, p_{n}$. However, it needs only the solution of a system of nonlinear equations, the dimension of which depends on $m$. The value of $m$ generally would be determined by the amount of computing power at our disposal, not by the number of points at which the functions were recorded. Therefore it is difficult to be prescriptive about $m$, although in numerical experiments we have found that the rankings are not very sensitive to choice of that quantity.

For instance, tilting the functional mean leads to solving a system of $m+2$ nonlinear equations 
in $C\left(t_{1}\right), \ldots, C\left(t_{m}\right), \lambda_{1}, \lambda_{2}$ :

$$
\begin{aligned}
\sum_{i=1}^{n} \exp \left\{\lambda_{1}-2 \lambda_{2} \sum_{k=1}^{m} C\left(t_{k}\right) X_{i}\left(t_{k}\right)\right\} X_{i}\left(t_{k}\right)-\bar{X}\left(t_{k}\right) & =C\left(t_{k}\right), \quad k=1, \ldots, m, \\
\sum_{k=1}^{m} C\left(t_{k}\right)^{2} & =\epsilon^{2}, \\
\sum_{i=1}^{n} \exp \left\{\lambda_{1}-2 \lambda_{2} \sum_{k=1}^{m} C\left(t_{k}\right) X_{i}\left(t_{k}\right)\right\} & =1,
\end{aligned}
$$

where $\lambda_{1}, \lambda_{2}$ are Lagrange multipliers, $C(t)=\bar{X}_{p}(t)-\bar{X}(t)$, and

$$
p_{i}=\exp \left\{\lambda_{1}-2 \lambda_{2} \sum_{k=1}^{m} C\left(t_{k}\right) X_{i}\left(t_{k}\right)\right\} \text {. }
$$

When $m=1$ the problem reduces to ranking univariate observations.

Similarly, tilting the functional covariance leads to solving a system of $\frac{1}{2} m(m+3)+2$ nonlinear equations in $C_{1}\left(t_{k}\right)$ for $k=1, \ldots, m$, and in $C_{2}\left(t_{k}, t_{\ell}\right)$ for $k, \ell=1, \ldots, m$, where, assuming that $C_{2}\left(t_{k}, t_{\ell}\right)=C_{2}\left(t_{\ell}, t_{k}\right)$, we impose the constraints

$$
\begin{aligned}
\sum_{i=1}^{n} p_{i} X_{i}\left(t_{k}\right) & =C_{1}\left(t_{k}\right), \quad k=1, \ldots, m, \\
\sum_{i=1}^{n} p_{i} X_{i}\left(t_{k}\right) X_{i}\left(t_{\ell}\right) & =C_{2}\left(t_{k}, t_{\ell}\right), \quad k, \ell=1, \ldots, m, \\
\sum_{k=1}^{m} \sum_{\ell=1}^{m}\left\{C_{2}\left(t_{k}, t_{\ell}\right)-C_{1}\left(t_{k}\right) C_{1}\left(t_{\ell}\right)-\widehat{K}\left(t_{k}, t_{\ell}\right)\right\}^{2} & =\epsilon^{2}, \quad \sum_{i=1}^{n} p_{i}=1 .
\end{aligned}
$$

Here the constraints are again imposed via Lagrange multipliers, $C_{1}(s)=\bar{X}_{p}(s), C_{2}(s, t)-$ $C_{1}(s) C_{1}(t)=\widehat{K}_{p}(s, t)$, and

$$
\begin{aligned}
p_{i}=\exp \left[\lambda_{1}-2 \lambda_{2} \sum_{k=1}^{m} \sum_{\ell=1}^{m}\{\right. & \left.C_{2}\left(t_{k}, t_{\ell}\right)-C_{1}\left(t_{k}\right) C_{1}\left(t_{\ell}\right)-\widehat{K}\left(t_{k}, t_{\ell}\right)\right\} \\
& \left.\times\left\{X_{i}\left(t_{k}\right)-C_{1}\left(t_{k}\right)\right\}\left\{X_{i}\left(t_{\ell}\right)-C_{1}\left(t_{\ell}\right)\right\}\right] .
\end{aligned}
$$

In the same manner, tilting the $j$ th eigenfunction requires solving a nonlinear system of 
$m+2$ equations, and tilting the conditional mean requires solving a nonlinear system of $m q+2$ equations, where $q$ is the number of values that $u$ can take.

As an illustration, we consider tilting the functional mean for various sample sizes $n$ and time-point numbers $m$. The original sample curves are generated from an outlier model (Sun and Genton, 2011): $X_{i}(t)=4 t+e_{i}(t)+\eta_{i} S_{i} L, i=1, \ldots, n, t \in[0,1]$, where $\eta_{i}=1$ with probability 0.1 and equals 0 with probability $0.9, L=6$ is the size of the contamination, $S_{i}$ is a sequence of random variables independent of $\eta_{i}$ taking values \pm 1 with probability $1 / 2$, and $e_{i}(t)$ is a Gaussian stochastic process with zero mean and exponential covariance function $K\left(t_{1}, t_{2}\right)=\exp \left(-\left|t_{2}-t_{1}\right|\right)$. All the computations are done in Matlab on a $2.80 \mathrm{GHz}$ Intel Xeon X5560 chip with 48GB of RAM. The computing times (in seconds) are reported in Table 1 . It can be seen that the tilting approach is feasible even for very large sample sizes $n$.

Table 1: Computing time (in seconds) for ranking functional data based on tilting the functional mean for various sample sizes $n$ and time points numbers $m$.

\begin{tabular}{|c|ccc|ccc|ccc|}
\hline \hline$n$ & \multicolumn{3}{|c|}{10,000} & \multicolumn{3}{c|}{100,000} & \multicolumn{3}{c|}{$1,000,000$} \\
\hline$m$ & 12 & 25 & 50 & 12 & 25 & 50 & 12 & 25 & 50 \\
\hline Tilting & 0.5 & 0.8 & 3.1 & 4.2 & 12.7 & 40.5 & 52.7 & 163.6 & 543.2 \\
\hline
\end{tabular}

\subsection{Sea Surface Temperature Data}

This dataset consists of monthly sea surface temperatures (SST) measured in degrees Celsius over the east-central tropical Pacific Ocean. In this case, each curve represents one year of observed SST from January 1951 to December 2007, and there are $n=57$ such curves.

We apply our tilting method with $\epsilon=0.2$ to this dataset, for tilting and ranking based on the functional mean and the functional covariance. The value 0.2 was determined simply by reducing $\epsilon$ a little more, for the sake of security, beyond the point where there was no further apparent change in the ranking. Choosing $\epsilon$ generally is simple and unsophisticated; it is unrelated to the 
problem of selecting a smoothing parameter, for example.

The results are presented in Figure 1, where the red curve represents the median, the dashed curve represents the mean, and the blue and green curves represent the lower $25 \%$ and upper $25 \%$ quantiles, respectively. The shading of the data curves corresponds to a ranking based on the weights $p_{i}$, from the highest ranked curve (i.e. the curve with index $\hat{\imath}_{1}(\epsilon)$ in $(15)$, indicated by light grey in the figure) to the lowest ranked one (indicated by dark grey).

The plots in the case of the sample mean, shown in the left hand panel of Figure 1, reveal that curves that have greatest influence (i.e. which correspond to small or large values of $p_{i}$ ) are those that are positioned relatively high up or low down the temperature axis. Moreover, the distribution of influential curves is quite asymmetric, with the least influential curves being more tightly bunched than the most influential ones. In particular, the lower $25 \%$ quantile curve
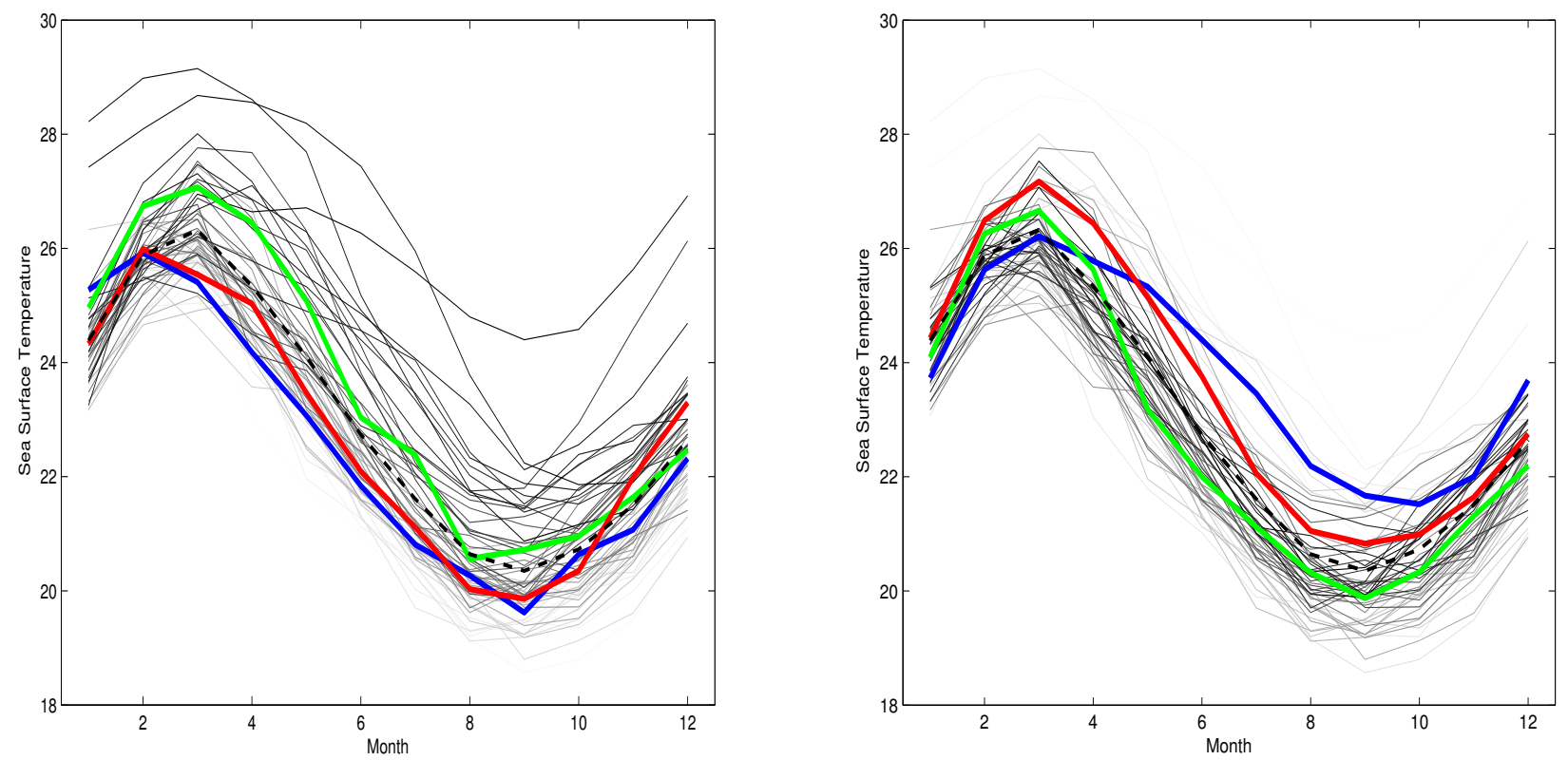

Figure 1: Tilting and ranking of sea surface temperature functional data (in degrees Celsius) with $\epsilon=0.2$ based on: functional mean (left panel) and functional covariance (right panel). The green, red and blue curves correspond to the upper 25\%, 50\% and lower 25\% quantiles of the weights, and the dashed curve represents the mean. 
is, on average, significantly closer to the median curve than is the upper $25 \%$ quantile curve. If these data were real valued then the distribution would be quite asymmetric, with one tail (say, the right tail) significantly longer than the other. In this case, moving from left to right on the real line, the lower $25 \%$ quantile, the median, the mean and the upper $25 \%$ quantile would be arranged in that order. This is also the order of those quantities in the left-hand panel of Figure 1, between months two and nine, and in this sense the influence of data curves on the mean exhibits a pattern that is familiar for real data drawn from a skewed distribution.

A ranking based on the functional mean is similar to that for real valued data in other respects, too. For example, the two darkest curves, i.e. the two high up most influential functions, correspond to the years 1983 and 1997 during which there was a so-called El Niño effect, the unusual warming of SST in the region where the data were gathered.

Of course, the data depictions in either panel of Figure 1 represent projections, shown in a two dimensional plot, of higher dimensional quantities, and so we should not expect them to always reflect the familiar "ordering" for real data. The right hand panel of Figure 1, which depicts the data functions in terms of their relative influence on functional covariance, is a case in point. There the functions representing upper and lower $25 \%$ quantiles lie on the same side, not opposite sides, of the median function throughout the first 5 months of the year, indicating that the above-mentioned projections do not, in the case of covariance, produce a separation that usefully grades the data from more to less influential. Variability through much of the year is seen to be relatively haphazard, in terms of its depiction in the right panel of Figure 1, with both low and high variability, relative to median variability, tending to be caused by functions which are relatively high on the temperature scale, whereas the "more average" curves (again in the sense of the median) are generally positioned towards relatively low temperatures.

The approach to functional ranking proposed in this article can be used to construct a func- 
tional boxplot as in Sun and Genton (2011, 2012a). Figure 2 depicts functional boxplots based on the functional mean ranking (left panel) and on modified band depth ranking (right panel). The general shape of the functional boxplot is similar for the two rankings, but the one based on the method in this paper detects one additional outlier corresponding to the year 1998, when the El Niño effect continued from 1997.

To provide further guidance in interpreting measures of influence for functional data we give, in Figure 3, results for scalar data. Specifically, the left panel of Figure 3 shows a plot of tilted weights in the case of the mean, $\bar{X}_{p}\left(t_{0}\right)=\sum_{i} p_{i} X_{i}\left(t_{0}\right)$, where $t=t_{0} \equiv$ month 7 . Here the results are easily interpreted; they are similar to those for the left panel of Figure 1, and in particular the median dot lies between the dots that represent lower and upper quantiles. (In both panels we use the colour and grey shade convention in Figure 1.) The right panel addresses the case of covariance, $\widehat{K}_{p}\left(t, t_{0}\right)$, viewed as a function of $t$. Here the interpretation reflects that for the right panel of Figure 1, where the median does lie between the $25 \%$ and $75 \%$ quantiles for month 7 .
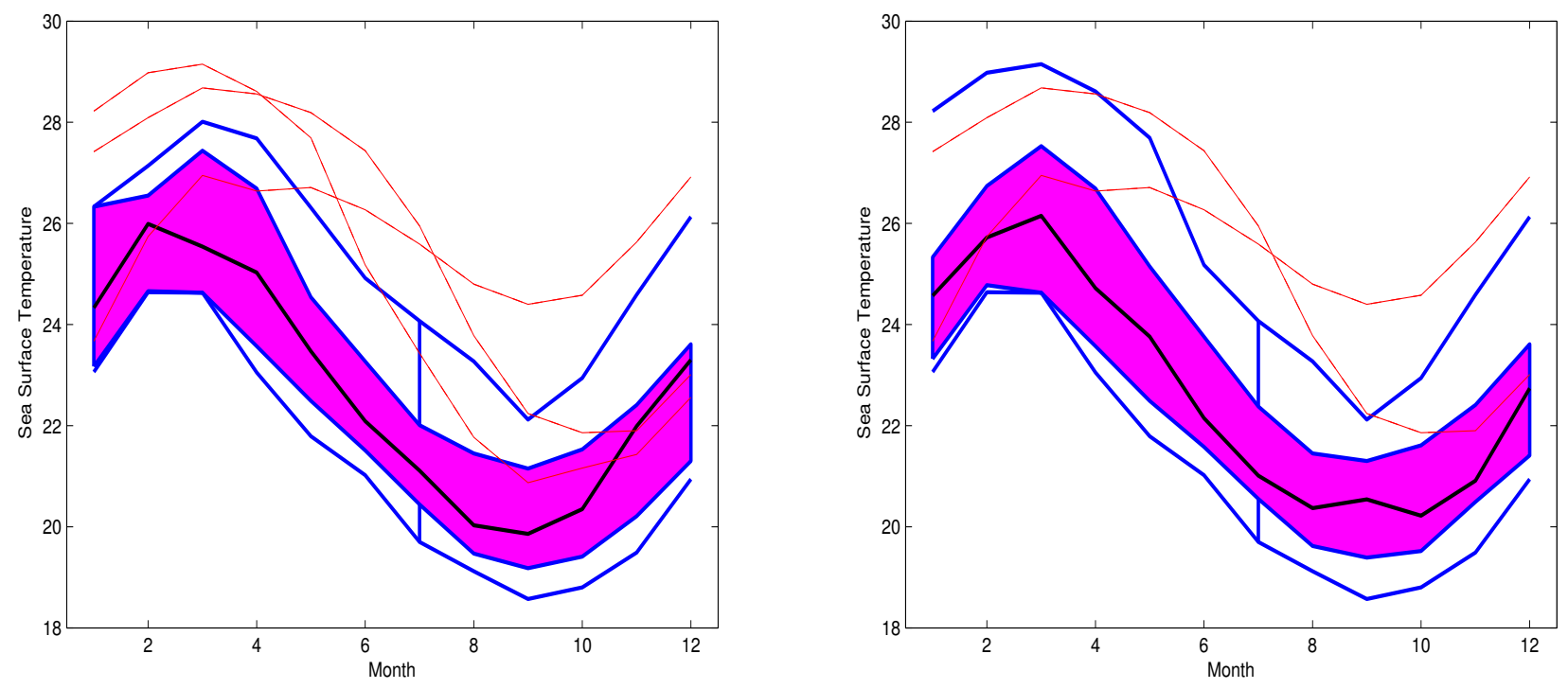

Figure 2: Functional boxplot of sea surface temperature functional data (in degrees Celsius) based on tilting and ranking the functional mean with $\epsilon=0.2$ (left panel). Functional boxplot of sea surface temperature functional data based on modified band depth (right panel). 

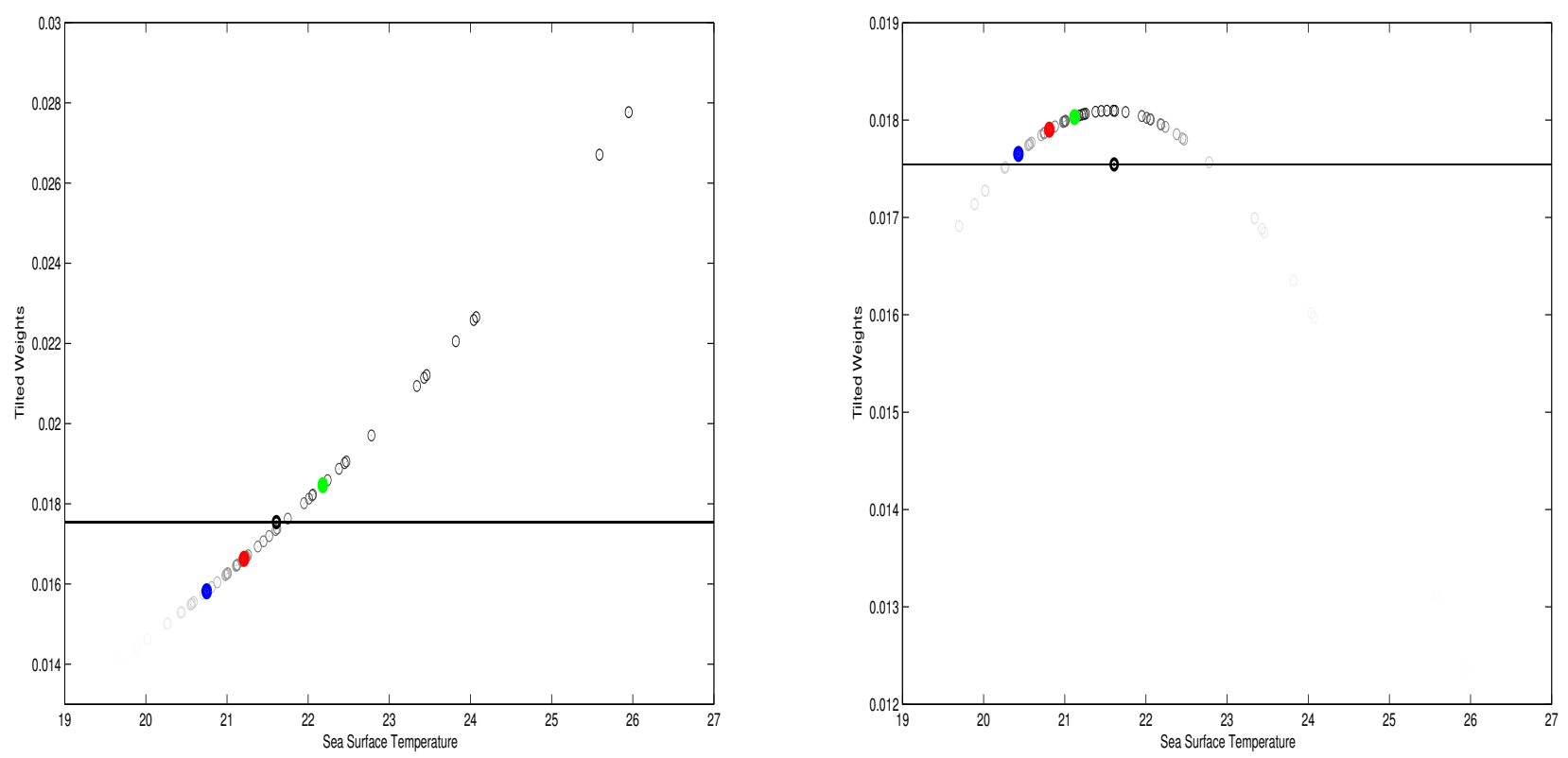

Figure 3: Tilting and ranking of univariate data with $\epsilon=0.2$ based on the functional mean $\bar{X}_{p}\left(t_{0}\right)$, where $t_{0}=$ month 7 (left panel), and functional covariance $\widehat{K}_{p}\left(t, t_{0}\right)$ (right panel). The green, red and blue points correspond to the upper $25 \%, 50 \%$ and lower $25 \%$ quantiles of the weights, and the black point represents the mean. The horizontal line represents the constant weight $1 / 57$.

In particular, as was also found in the context of Figure 1, the data projection represented by the right panel of Figure 3 does order the data in an informative way for month 7 .

Next, in Figure 4 (left hand panel) we visualize the weights $p_{i}$, for $i=1, \ldots, 57$, based on tilting the functional mean, when $d_{1}(p)$ is constrained as at (13). In particular, the values of $p_{i}$ are now functions of $\epsilon>0$. The vertical black line corresponds to $\epsilon=0.2$. The green, red and blue curves correspond to the upper $25 \%, 50 \%$ and lower $25 \%$ quantiles of the weights. As anticipated by the theorem in Section 2.3, the ranking of the weights remains the same as $\epsilon \downarrow 0$. In order to further interpret the weights we investigate the empirical influence of one outlying observation. We take that curve to be the median function, represented in red in the left hand panel of Figure 1 , and we perturb that observation, $X_{28}(t)$, at a single time point, $t=6$, representing June, by an additive quantity $\xi$. That is, we consider the function $X_{28}(6)+\xi$. In the right hand panel 



Figure 4: Left: Weights $p_{i}$, for $i=1, \ldots, 57$, based on tilting the functional mean and associated with each sea surface temperature curve. The $p_{i}$ s are viewed as functions of $\epsilon$. The vertical black line corresponds to $\epsilon=0.2$. The green, red and blue curves correspond to the upper $25 \%, 50 \%$ and lower $25 \%$ quantiles of the weights. Right: Weights $p_{i}$, for $i=1, \ldots, 57$, based on tilting the functional mean with $\epsilon=0.2$ and associated with each sea surface temperature curve viewed as a function of $X_{28}(6)+\xi$. The vertical blue line corresponds to $\xi=0$ (no contamination). The red dashed curve depicts the change in the weight $p_{28}$ when the curve $X_{28}(t)$ is perturbed by adding $\xi$.

of Figure 4 we plot the weights $p_{i}$, for $i=1, \ldots, 57$, based on tilting the functional mean with $\epsilon=0.2$ and associated with each sea surface temperature observation viewed as a function of $X_{28}(6)+\xi$. The vertical blue line corresponds to $\xi=0$ (no contamination). The red dashed curve depicts the change in the weight $p_{28}$ when the functional observation $X_{28}(t)$ is perturbed to $X_{28}(6)+\xi$. We see that $p_{i}$ becomes larger or smaller as the positive or negative amount of the contamination $\xi$ changes in the same way, and eventually becomes the largest or smallest weight among those for the functional sample.

\subsection{Simulation Comparison}

In order to compare the tilting approach to ranking with the band depth ranking, we perform the following simulation experiment. We simulate $n=100$ functional curves, each observed at 
12 time points, with mean zero unit variance Gaussian distribution and with an exponential autocovariance function $\gamma(h)=\exp (-h / 0.001)$. We then find the median curve from this sample with the tilting approach and with the band depth approach. We repeat this experiment 1000 times. Hence, we have 1000 medians from tilting and 1000 medians from band depth. Figure 5 summarizes this information by means of functional boxplots. It can be seen that the medians obtained by tilting and the medians obtained by band depth have approximately similar distribution patterns.
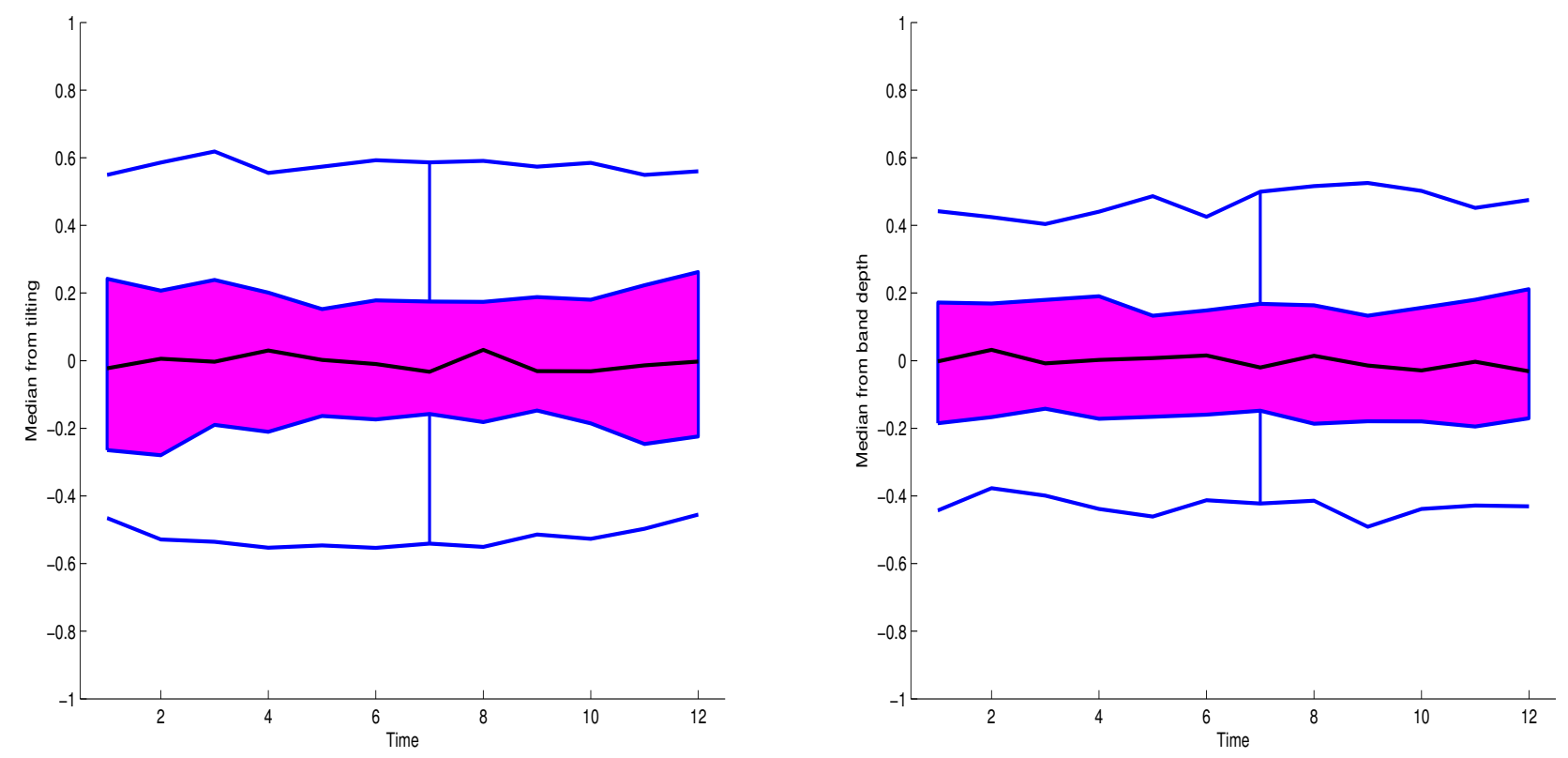

Figure 5: Functional boxplots of 1000 medians obtained by tilting (left panel) and band depth (right panel).

\subsection{Child Growth Data}

This dataset consists of the heights, in centimetres, of 39 boys and 54 girls, measured at 31 unequally spaced ages from 1 year to 18 years; see Ramsay and Silverman (2005) for details. We consider the boys and girls to come from separate populations. Within each population the growth curves have similar shape, and in particular are monotone increasing. Therefore it is 
not straightforward to identity unusual growth patterns. We shall first determine and discuss the rankings of these curves, using our tilting approach, and then we shall compare the level of information that can be extracted from the data using functional boxplots, based on rankings provided by tilting or by modified band depth (López-Pintado and Romo, 2009), respectively.

First we apply our tilting method, using the functional data sample mean as the statistic of interest. The results are presented in Figure 6, where the red curve represents the median, the dashed curve depicts the mean, and the blue and green curves show the lower $25 \%$ and upper $25 \%$ quantiles, respectively. The shading of the data curves corresponds to a ranking based on the weights $p_{i}$, from the highest ranked curve (i.e. the curve with index $\hat{\imath}_{1}(\epsilon)$ in $(15)$, indicated by light grey in the figure) to the lowest ranked one (indicated by dark grey). The plots reveal that curves that have greatest influence (i.e. which correspond to small or large values of $p_{i}$ ) are
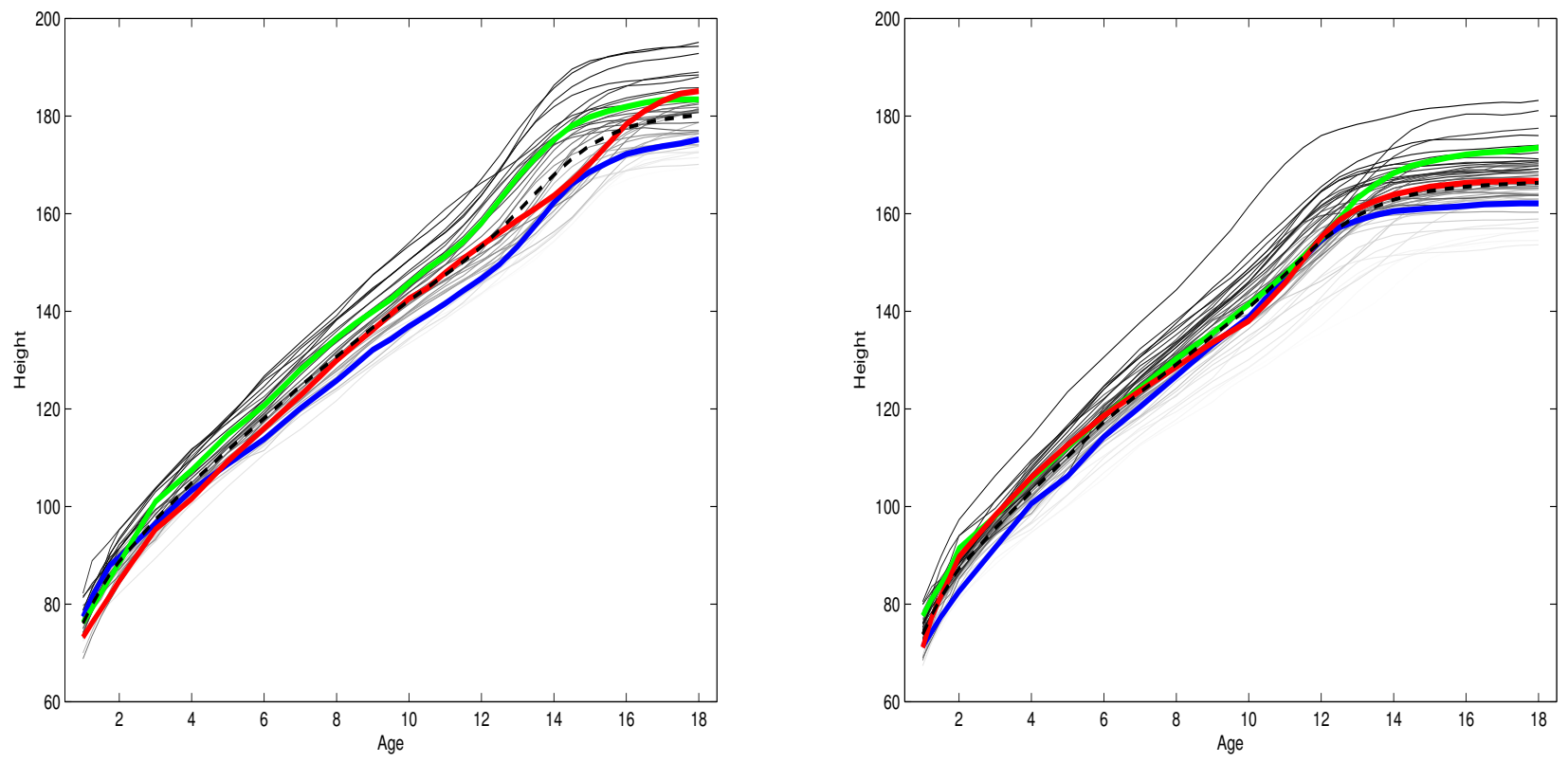

Figure 6: Tilting and ranking of child growth data, using the functional data sample mean as the statistic of interest and in the case of boys (left panel) and girls (right panel). The green, red and blue curves correspond respectively to the upper $25 \%, 50 \%$ and lower $25 \%$ quantiles of the weights, and the dashed curve represents the mean. 

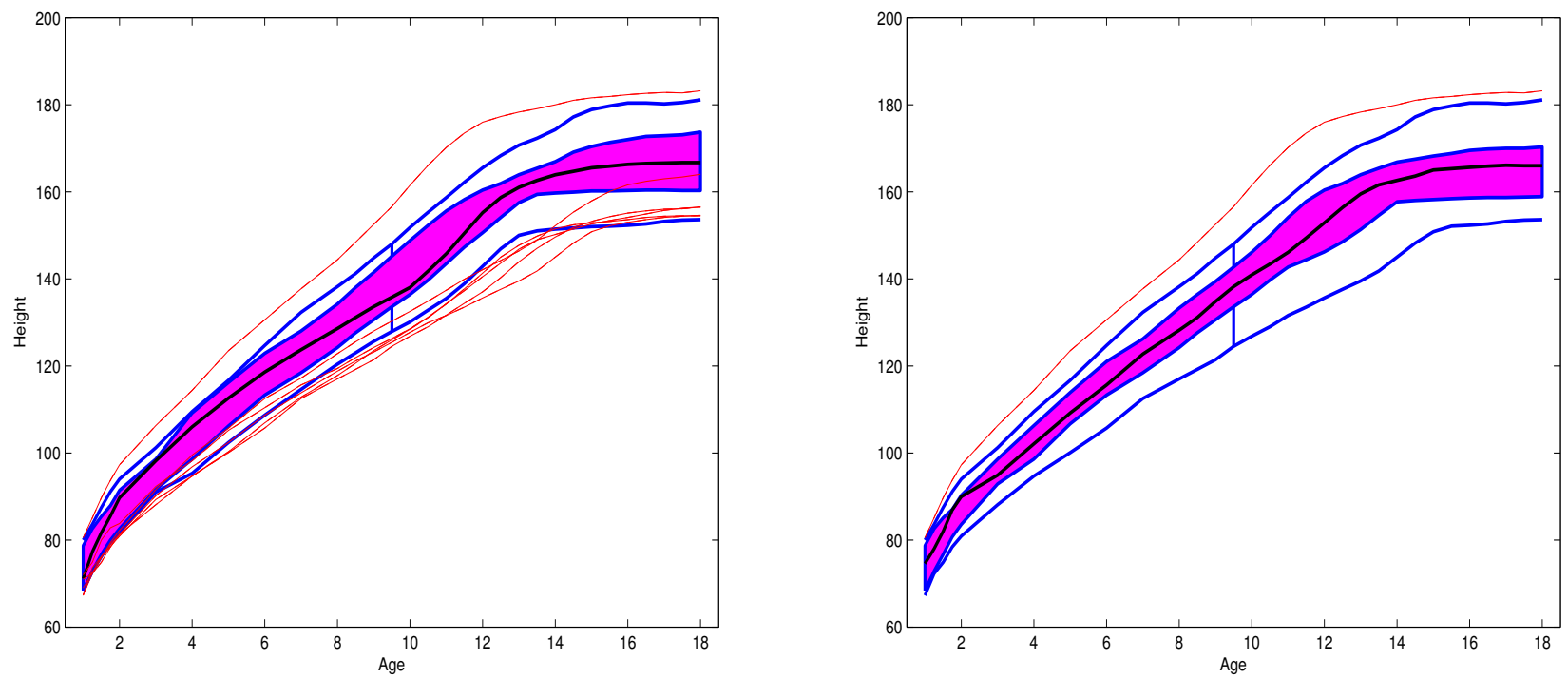

Figure 7: Functional boxplot of child growth data for the sample of girls, based on tilting and using the functional data sample mean as the statistic of interest (left panel), and based on modified band depth (right panel).

positioned relatively high up or low down the height axis. The distribution of influential curves is more symmetric in this example than in the example treated in Section 3.2.

Our approach to functional data ranking can also be used to construct functional boxplots, as in Sun and Genton (2011, 2012a). Figure 7 depicts, for the girls, functional boxplots based on the functional mean ranking (left panel) and on modified band depth ranking (right panel). The general shape of the functional boxplot is similar for the two rankings, but that based on the method suggested in this paper detects five additional outliers, corresponding to girls with very low growth curves, especially in their teenage years. For the boys, the two functional boxplots are similar in shape and neither approach detects outlying data curves. This is a classic, benchmark dataset, and readers seeking further discussion of the data will find it in Ramsay and Silverman (2005); see particularly pages 1, 62, 88, 112 and 165. 


\section{Appendix: Proof of the Theorem}

Assumption (17) implies that, if $p$ is an extremum of

$$
\int\left(\widehat{\omega}_{p}-\widehat{\omega}\right)^{2}+\lambda_{1} \sum_{i=1}^{n} p_{i} \log p_{i}+\lambda_{2}\left(\sum_{i=1}^{n} p_{i}-1\right)
$$

where $\lambda_{1}$ and $\lambda_{2}$ denote Lagrange multipliers, then

$$
\begin{aligned}
p_{j} & =\exp \left\{\lambda_{3}+\lambda_{4} \sum_{i=1}^{n}\left(p_{i}-n^{-1}\right) \int \Delta_{i} \Delta_{j}+\lambda_{4} A_{j}+O\left(\epsilon^{3}\right)\right\} \\
& =\frac{1}{n}\left\{\lambda_{5}+\lambda_{4} \int \psi \Delta_{j}+\lambda_{4} A_{j}+\frac{1}{2}\left(\lambda_{4} \int \psi \Delta_{j}\right)^{2}+O\left(\epsilon^{3}\right)\right\} .
\end{aligned}
$$

Here and below, $\lambda_{1}, \ldots, \lambda_{5}$ do not depend on $p$, and we have defined

$$
\psi=\sum_{i=1}^{n}\left(p_{i}-n^{-1}\right) \Delta_{i}
$$

equal to the dominant term on the right hand side of (16). The property $\Delta_{i}=O(\epsilon)$ in $(18)$ implies that $\psi=O(\epsilon)$.

Using (18), and the fact that $\sum_{j} p_{j}=1$, it can be deduced from (A.1) that, for each $j$,

$$
p_{j}=\frac{1}{n}\left\{1+\lambda_{4} \int \psi \Delta_{j}+\lambda_{4} A_{j}+\frac{1}{2} \lambda_{4}^{2} S_{j}+O\left(\epsilon^{3}\right)\right\}
$$

where

$$
S_{j}=\left(\int \psi \Delta_{j}\right)^{2}-\frac{1}{n} \sum_{i=1}^{n}\left(\int \psi \Delta_{i}\right)^{2} .
$$


Therefore, again using (18),

$$
\begin{aligned}
\sum_{j=1}^{n} p_{j} \log \left(n p_{j}\right)= & \frac{1}{n} \sum_{j=1}^{n}\left\{1+\lambda_{4} \int \psi \Delta_{j}+\lambda_{4} A_{j}+\frac{1}{2} \lambda_{4}^{2} S_{j}\right\} \\
& \times\left\{\lambda_{4} \int \psi \Delta_{j}+\lambda_{4} A_{j}+\frac{1}{2} \lambda_{4}^{2} S_{j}-\frac{1}{2}\left(\lambda_{4} \int \psi \Delta_{j}\right)^{2}\right\}+O\left(\epsilon^{3}\right) \\
= & \frac{1}{2} \sum_{j=1}^{n}\left(\lambda_{4} \int \psi \Delta_{j}\right)^{2}+O\left(\epsilon^{3}\right) .
\end{aligned}
$$

Furthermore, in view of (16) and (A.2),

$$
\int\left(\widehat{\omega}_{p}-\widehat{\omega}\right)^{2}=\int \psi^{2}+O\left(\epsilon^{3}\right) .
$$

Hence, the algorithm chooses $p$ (and therefore $\psi$ ) to minimise

$$
\sum_{j=1}^{n}\left(\lambda_{4} \int \psi \Delta_{j}\right)^{2}+O\left(\epsilon^{3}\right)
$$

or equivalently, to minimise

$$
\lambda_{4}^{2} \int \psi(\widehat{M} \psi)+O\left(\epsilon^{3}\right)
$$

subject to

$$
\int \psi^{2}=\epsilon^{2}+O\left(\epsilon^{3}\right)
$$

Since $p_{j}$ satisfies (A.3) then

$$
\begin{aligned}
\psi & =\sum_{i=1}^{n}\left(p_{i}-n^{-1}\right) \Delta_{i}=\frac{1}{n} \sum_{i=1}^{n}\left(\lambda_{4} \int \psi \Delta_{i}+\lambda_{4} A_{i}+\frac{1}{2} \lambda_{4}^{2} S_{i}\right) \Delta_{i}+O\left(\epsilon^{3}\right) \\
& =\lambda_{4} \frac{1}{n} \sum_{i=1}^{n} \Delta_{i} \int \psi \Delta_{i}+O\left(\epsilon^{3}\right)=\lambda_{4} \widehat{M} \psi+O\left(\epsilon^{3}\right) .
\end{aligned}
$$

Therefore, up to an error that is negligible as $\epsilon \downarrow 0, \psi$ is an eigenfunction of $\widehat{M}$ with eigenvalue $\lambda_{4}^{-1}$. Hence, minimising the quantity at (A.4) is equivalent to minimising $\lambda_{4} \int \psi^{2}+O\left(\epsilon^{3}\right)$, which, we know from (A.5), is equivalent to minimising $\lambda_{4} \epsilon^{2}+O\left(\epsilon^{3}\right)$. Hence, $\lambda_{4}$ should be as small as 
possible, and so $\psi$ should equal the first-ranked eigenvector of $\widehat{M}$, which in turn equals $\hat{\beta}_{1}$, plus a term of order $\epsilon$, and $\lambda_{4}=\hat{\alpha}_{1}^{-1}+O(\epsilon)$. These results and (A.3) together imply that the ordering of indices $\hat{\imath}_{1}, \ldots, \hat{\imath}_{p}$ corresponding to $p_{\hat{\imath}_{1}}(\epsilon)>\ldots>p_{\hat{\imath}_{n}}(\epsilon)$, for all sufficiently small $\epsilon$, is identical to the ordering at $(23)$.

\section{References}

Bravo, F. (2005), "Blockwise empirical entropy tests for time series regressions," Journal of Time Series Analysis, 26, 185-210.

Camponovo, L., and Otsu, T. (2012), "Breakdown point theory for implied probability bootstrap," Econometrics Journal, 15, 32-55.

Choi, E., Hall, P., and Presnell, B. (2000), "Rendering parametric procedures more robust by empirically tilting the model," Biometrika, 87, 453-465.

Critchley, F., and Marriott, P. (2004), "Data-informed influence analysis," Biometrika, 91, 124140.

Critchley, F., Atkinson, R. A., Lu, G., and Biazi, E. (2001), "Influence analysis based on the case sensitivity function," Journal of the Royal Statistical Society Series B, 63, 307-323.

Cressie, N. A. C., and Read, T. R. C. (1984), "Multinomial goodness-of-fit tests," Journal of the Royal Statistical Society Series B, 46, 440-464.

Ferraty, F., and Vieu, P. (2006), Nonparametric Functional Data Analysis: Theory and Practice, New York: Springer.

Genton, M. G., and Ruiz-Gazen, A. (2010), "Visualizing influential observations in dependent data," Journal of Computational and Graphical Statistics, 19, 808-825. 
Genton, M. G., Johnson, C., Potter, K., Stenchikov, G., and Sun, Y. (2014), "Surface boxplots," Stat, 3, 1-11.

Hall, P., and Presnell, B. (1999a), "Intentionally biased bootstrap methods," Journal of the Royal Statistical Society Series B, 61, 143-158.

Hall, P., and Presnell, B. (1999b), "Biased bootstrap methods for reducing the effects of contamination," Journal of the Royal Statistical Society Series B, 61, 661-680.

Hall, P., Titterington, D. M., and Xue, J.-H. (2009), "Tilting methods for assessing the influence of components in a classifier," Journal of the Royal Statistical Society Series B, 71, 783-803.

Hall, P., and Yao, Q. (2003), "Data tilting for time series," Journal of the Royal Statistical Society Series B, 65, 425-442.

Hyndman, R. J., and Shang, H. L. (2010), "Rainbow plots, bagplots, and boxplots for functional data," Journal of Computational and Graphical Statistics, 19, 29-45.

Lazar, N. A. (2005), "Assessing the effect of individual data points on inference from empirical likelihood," Journal of Computational and Graphical Statistics, 14, 626-642.

López-Pintado, S., and Romo, J. (2009), "On the concept of depth for functional data," Journal of the American Statistical Association, 104, 718-734.

López-Pintado, S., Sun, Y., Lin, J. K., and Genton, M. G. (2014), "Simplicial band depth for multivariate functional data," Advances in Data Analysis and Classification, 8, 321-338.

Ramsay, J. O., Hooker, G., and Graves, S. (2009), Functional Data Analysis with $R$ and MAT$L A B$, New York: Springer Verlag.

Ramsay, J. O., and Silverman, B. W. (2005), Functional Data Analysis (2nd ed.), New York: Springer Verlag. 
Sun, Y., and Genton, M. G. (2011), "Functional boxplots," Journal of Computational and Graphical Statistics, 20, 313-334.

Sun, Y., and Genton, M. G. (2012a), "Adjusted functional boxplots for spatio-temporal data visualization and outlier detection," Environmetrics, 23, 54-64.

Sun, Y., and Genton, M. G. (2012b), "Functional median polish," Journal of Agricultural, Biological, and Environmental Statistics, 17, 354-376.

Sun, Y., Genton, M. G., and Nychka, D. W. (2012), "Exact fast computation of band depth for large functional datasets," Stat, 1, 68-74.

Xu, K. L., and Phillips, P. C. B. (2011), "Tilted nonparametric estimation of volatility functions with empirical applications," Journal of Business and Economic Statistics, 29, 518-528.

Yu, G., Zou, C., and Wang, Z. (2012), "Outlier detection in functional observations with applications to profile monitoring," Technometrics, 54, 308-318. 